\title{
An Iterative Learning Control Design for Self-Servowriting in Hard Disk Drives
}

\author{
Shang-Chen $\mathbf{W u}^{*}$ Masayoshi Tomizuka ${ }^{* *}$ \\ * Department of Mechanical Engineering, University of California at \\ Berkeley, Berkeley, CA 94720,USA (e-mail: scwu@berkeley.edu) \\ ** Department of Mechanical Engineering, University of California at \\ Berkeley, Berkeley, CA 94720, USA (e-mail: \\ tomizuka@me.berkeley.edu)
}

\begin{abstract}
This paper considers the modeling and compensator design for Self-ServoWriting (SSW) in disk drives. An Iterative Learning Control (ILC) based scheme is established to deal with radial error propagation and improve the quality of written tracks. In the proposed scheme, a feedback controller for track following is first designed to achieve good disturbance attenuation. Then, an ILC structure is applied to generate an external signal, which is injected into the feedback loop in order to compensate for the written-in errors in the previous track while the next track is written. As a result, the error propagation can be contained. The learning controller is synthesized by solving Linear Matrix Inequality (LMI) equations to ensure the stability and monotonic convergence of the control algorithm. Simulation results show the effectiveness of the proposed scheme on the error containment which results in good quality written tracks.
\end{abstract}

\section{INTRODUCTION}

In the sector servo system for Hard Disk Drives (HDDs), a circular disk is divided into equally sized angular pieces, called servo sectors. Laid on the boundaries of the servo sectors are servo fields on which special servo patterns are embedded. Whenever the Read/Write head (R/W head) passes over the servo patterns, a waveform is read back and decoded to generate a position error signal (PES) which indicates the deviation of the $\mathrm{R} / \mathrm{W}$ head from the center of the track. PES is then utilized for the control of the head position. With the continuously increasing track density, the required accuracy on the placement of servo patterns is proportionally increased and becomes a crucial issue.

Servowriting is the process to write servo patterns onto the disk. The goal is to place the servo patterns in centric circles with minimum variation of track spacing for all tracks. Conventionally, the process is accomplished by a high precision device called a servowriter, which uses external positional references to accurately position the heads in the disk drive for writing the servo patterns. During the process, in order for the device to access the heads, the drive cover has to be removed. Hence, a clean-room environment is required to servowrite a disk drive. With nowadays high density drives, the servowrting process is extremely time consuming. Consequently, long hours spent on expensive servowriters in clean-room space result in substantial manufacturing cost. Recently, a technique called Self-ServoWriting (SSW) has been developed in order to cut down the cost.

For one type of SSW process (Cribbs et al. [1995]), the basic concept is to propagate the servo patterns from a

\footnotetext{
* This research was supported by the Computer Mechanics Laboratory (CML) in the Department of Mechanical Engineering, University of California at Berkeley.
}

few seed tracks by the drives itself. Only the seed tracks are written by an external device. Thereafter, the majority portion of the servowriting process is done after the drive is assembled. The time spent in the clean room and thus the manufacturing cost can be dramatically reduced. However, in SSW, an absolute position reference is not available. Instead, a previously written track is used as a relative position reference. As a result, the written-in error may be re-produced and even amplified when successive tracks are written. The phenomenon is called Radial Error Propagation, the avoidance of which is the major challenge in SSW. Therefore, the motivation of this research is to prevent radial error propagation and improve the quality of tracks in terms of written-in errors.

An Iterative Learning Control (ILC) based control structure can be an effective approach to mitigate radial error propagation. Some researchers (see Ye et al. [2002], Melkote and McNab [2006]) have formulated the problem into a similar manner and provided heuristic design methods. In this paper, we propose an ILC based scheme and establish a systematic framework for the compensator design. The learning controller in the ILC structure is synthesized by solving Linear Matrix Inequalities (LMIs) to ensure the stability and monotonic convergence of the control algorithm. The disturbance and noise rejection is also taken into consideration. The remainder of this paper is organized as follows. In Section 2, the modeling of the SSW process and the root cause of radial error propagation are briefly reviewed. Then Section 3 describes the proposed ILC scheme. LMI conditions are derived for controller synthesis. In Section 4, the proposed method is applied to a HDD benchmark problem. Finally, conclusions are given in Section 5 . 


\section{PROBLEM FORMULATION}

\subsection{Modeling of the SSW Process}

Consider a simplified setting for a SSW process, which contains the following steps:

(1) A seed track which may be written by a servo track writer is available on the disk. PES signal can be obtained when the read head is track-following on the seed track.

(2) Assume a constant read-head-to-write-head offset of one track width. Make the read head follows the seed track in the usual track following mode while the write head writes servo patterns for the next track.

(3) The read head uses the newly written track as a following reference while the write head writes the next track.

(4) Continue Step (3) until all tracks are written.

Figure 1 illustrates the described SSW process, where $y_{i}(k)$ represents the track profile of track $i$ at servo sector $k$. In other words, its value indicates the deviation of the servo written track center from the perfect circular track center. It is the so called Written-In Error or Written-In Repeatable RunOut (WIRRO). A block diagram of the SSW system is shown in Fig. 2. The system contains a standard track following servo loop with Voice Coil Motor as plant $P(z)$ and the feedback controller $C(z)$. The read head follows on track $i$ with track profile $y_{i}(k)$ as the reference and generates PES signal for feedback control. The plant output is the write head position where the write head places the servo patterns for the next track and generate the track profile $y_{i+1}(k)$. Notice that $y_{i+1}(k)$ is related to $y_{i}(k)$ by the complimentary sensitivity function $T(z)=P(z) C(z) /[1+P(z) C(z)]$, i.e.,

$$
y_{i+1}(k)=T(z) y_{i}(k)
$$

Equation (1) governs the evolution of the track profile for every servowriting step.

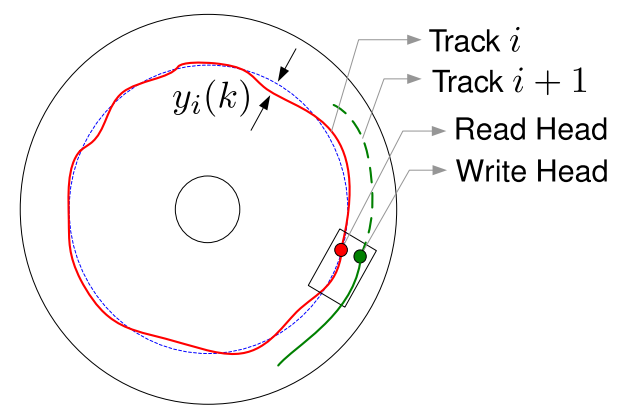

Fig. 1. An illustration of Self-Servowriting process

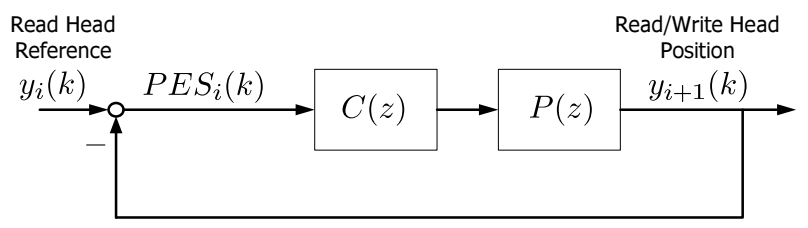

Fig. 2. Block diagram of Self-Servowriting servo loop

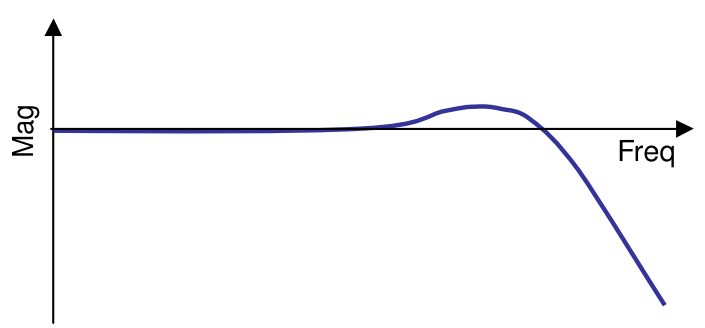

Fig. 3. A typical complimentary sensitivity function for HDD servo systems

\subsection{Radial Error Propagation}

In an ideal situation, if $T(z)=1$, then from $(1), y_{i+1}(k)$ will be the exact copy of $y_{i}(k)$, which means every written tracks will have equal spacing which is the desired one track width. However, in the actual case, the gain of $T(z)$ must be larger than 1 at some frequencies. The compound effect causes the written-in error build up in the radial direction across the disk. The root cause of the radial error propagation is due to the integral constraint on the complimentary sensitivity function. According to Bode's integral theorem, given a stable plant $P(z)$ and a stable controller $C(z)$ such that open loop transfer function $P(z) C(z)$ has relative degree larger or equal to two, the integral of the log magnitude of the sensitivity function over the entire frequency from zero to infinity must be equal to zero. In most cases, the HDD servo design usually satisfies the above condition (Al-Mamun et al. [2007]). Furthermore, as an extension of the theorem, the complimentary sensitivity function of the system has to obey the same control limitation. A typical complimentary sensitivity function for a HDD servo system is shown in Fig. 3. It is observed that less than unity gain is required at high frequencies for noise attenuation. Therefore, the complimentary sensitivity hump appears inevitable due to the zero integral constraint. Since the gain of $T(z)$ is larger than one at certain frequencies, according to (1), the frequency components of $y_{i}(k)$ may be amplified and transferred to $y_{i+1}(k)$. In addition, external disturbances and noise could cause extra off-track motion on the $\mathrm{R} / \mathrm{W}$ head. As a result, compound errors propagate across the tracks and lead to excessive track non-circularity.

\section{ILC COMPENSATION SCHEME}

To prevent radial error propagation, one way is to inject a compensation signal $u_{i}(k)$ as shown in Fig 4. Ideally, if $u_{i}(k)=y_{i}(k)$, then $y_{i+1}(k)=0$ may be possible. That means zero WIRRO in the next track is achievable when the WIRRO in the current track is completely cancelled out. In practice, track profile $y_{i}(k)$ is not available. However, the error signal from the previous track, $e_{i-1}(k)$, is measurable and can be utilized to estimate $y_{i}(k)$. If it is considered as an iteration every time a new track is written, then the concept can be expressed as an Iterative Learning Control law:

$$
u_{i}(k)=F(z) e_{i-1}(k) .
$$

The compensation signal $u_{i}(k)$ is generated by putting $e_{i-1}(k)$ through a learning filter $F(z)$ in every iteration.

Now the evolution equation for track profiles can be derived according to Fig. 4 as follows. 


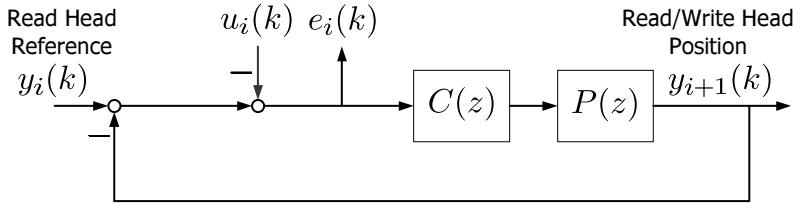

Fig. 4. Block diagram of SSW servo loop with external compensation signal $u_{i}(k)$

$$
\begin{aligned}
y_{i+1}(k) & =T(z) y_{i}(k)-T(z) u_{i}(k) \\
& =T(z) y_{i}(k)-T(z) F(z) e_{i-1}(k) \\
& =T(z) y_{i}(k)-T(z) F(z)(P(z) C(z))^{-1} y_{i}(k) \\
y_{i+1}(k) & =[T(z)-S(z) F(z)] y_{i}(k),
\end{aligned}
$$

where $S(z)=1 /(1+P(z) C(z))$ is the sensitivity function. Notices that $[T(z)-S(z) F(z)]$ becomes the essential transfer function relating the current and the next track profiles. In the next section, the evolution equation is transformed to the lifted domain for design purpose.

\subsection{Design in the Lifted Domain}

Using the impulse response representation, the sensitivity function $S(z)$ is written as

$$
S(z)=s_{0}+s_{1} z^{-1}+s_{2} z^{-2}+\cdots+s_{N-1} z^{-N+1},
$$

where $N$ denotes the number of servo sectors. The impulse response values, $s_{0}, \cdots, s_{N-1}$, are sometimes called the Markov parameters of $S(z)$. Construct an $N$-by- $N$ matrix S using $s_{0}, \cdots, s_{N-1}$ as follows.

$$
\mathbf{S}=\left[\begin{array}{cccc}
s_{0} & 0 & \ldots & 0 \\
s_{1} & s_{0} & \ldots & 0 \\
\vdots & \vdots & \ddots & \vdots \\
s_{N-1} & s_{N-2} & \ldots & s_{0}
\end{array}\right], \quad \mathbf{T}=\left[\begin{array}{cccc}
t_{0} & 0 & \ldots & 0 \\
t_{1} & t_{0} & \ldots & 0 \\
\vdots & \vdots & \ddots & \vdots \\
t_{N-1} & t_{N-2} & \ldots & t_{0}
\end{array}\right]
$$

Notice that $\mathbf{T}$ is constructed in the similar manner from the transfer function $T(z)$. Then, from (3) and (5), one can convert the SSW process with ILC into a Lifted System,

$$
\left[\begin{array}{c}
y_{i+1}(0) \\
y_{i+1}(1) \\
\vdots \\
y_{i+1}(N-1)
\end{array}\right]=[\mathbf{T}-\mathbf{S F}]_{N \times N}\left[\begin{array}{c}
y_{i}(0) \\
y_{i}(1) \\
\vdots \\
y_{i}(N-1)
\end{array}\right]
$$

where the input and the output are adjacent track profiles in the vector form. $\mathbf{F}$ with its matrix elements related to the learning filter $F(z)$ is the design parameter. The objective is to find a $\mathbf{F}$ such that $y_{i}$ converges to zero.

The system in (6) is analogous to a standard discrete time system, $x_{k+1}=A x_{k}$. Hence, it is easy to see that the convergence condition for the lifted system (6) is governed by the radius of the matrix $(\mathbf{T}-\mathbf{S F})$, i.e.,

$$
\begin{aligned}
& \lim _{i \rightarrow \infty} y_{i}(k)=0 \quad \text { for } k=0,1, \cdots, N-1 \\
& \Longleftrightarrow|\lambda(\mathbf{T}-\mathbf{S F})|<1
\end{aligned}
$$

However, for the SSW application, it is desirable to have $\left\|y_{i}\right\|_{2}$ keep deceasing with each iteration in some sense. This is called Monotonic Decreasing, which requires a stronger condition (see Bristow et al. [2006]):

$$
\begin{aligned}
& \lim _{i \rightarrow \infty} y_{i}(k)=0 \quad \text { and } \quad\left\|y_{i+1}(k)\right\|_{2}<\left\|y_{i}(k)\right\|_{2} \\
& \Longleftrightarrow\|\mathbf{T}-\mathbf{S F}\|_{2}=\bar{\sigma}(\mathbf{T}-\mathbf{S F})<1
\end{aligned}
$$

Notice that $\|\cdot\|_{2}$ denotes the spectral norm (maximum singular value). It is known that given a matrix $A(x)$ with a variable $x,\|A(x)\|_{2}$ is a convex function of $x$. In addition, the condition (8) can be re-formulated as (see Boyd and Vandenberghe [2004]),

$$
\begin{aligned}
& \bar{\sigma}(\mathbf{T}-\mathbf{S F})=\sqrt{\lambda_{\max }\left[(\mathbf{T}-\mathbf{S F})(\mathbf{T}-\mathbf{S F})^{T}\right]}<1 \\
\Longleftrightarrow & \mathbf{I}_{N \times N}-\left[(\mathbf{T}-\mathbf{S F})(\mathbf{T}-\mathbf{S F})^{T}\right]>0 \\
\Longleftrightarrow & {\left[\begin{array}{cc}
\mathbf{I}_{N \times N} & \mathbf{T}-\mathbf{S F} \\
(\mathbf{T}-\mathbf{S F})^{T} & \mathbf{I}_{N \times N}
\end{array}\right]>0 }
\end{aligned}
$$

As a result, the design of $\mathbf{F}$ satisfying (8) is equivalent to solving a LMI feasibility problem (9). The design matrix $\mathbf{F}$ could be a full matrix. However, a large amount of memory units are required to store the filter parameters. In practical applications, $\mathbf{F}$ could take the following form with constant diagonal terms,

$$
\mathbf{F}=\left[\begin{array}{cccccc}
f_{0} & f_{1}^{N} & f_{2}^{N} & & & \\
f_{1}^{C} & f_{0} & f_{1}^{N} & f_{2}^{N} & & \\
f_{2}^{C} & f_{1}^{C} & f_{0} & f_{1}^{N} & \ldots & \\
& f_{2}^{C} & f_{1}^{C} & f_{0} & \ldots & f_{2}^{N} \\
& & \vdots & \vdots & \ddots & f_{1}^{N} \\
& & & f_{2}^{C} & f_{1}^{C} & f_{0}
\end{array}\right]_{N \times N}
$$

corresponding to a Linear Time Invariant filter $F(z)$ with Finite Impulse Response (FIR),

$$
F(z)=\cdots+f_{2}^{N} z^{2}+f_{1}^{N} z^{1}+f_{0}+f_{1}^{C} z^{-1}+f_{2}^{C} z^{-2}+\cdots
$$

Notice that the filter taps with coefficients $f_{l}^{N}, l \in \mathbb{N}$, represent the time-advance terms. In other words, they are Non-Causal terms. According to (2), the error signal from previous iteration is recorded. That means $e_{i-1}(k)$ $k=0, \cdots, N-1$ is available for control computation. Therefore, a non-causal $F(z)$ is realizable with any nonzero coefficients $f_{l}^{N}$. In the proposed design method, special treatment is not required for the non-causal design. Once the number of non-zero coefficients is decided, the matrix $\mathbf{F}$ in (9) can be solved by a standard LMI solver (Sturm et al. [2006]).

\subsection{Disturbance and Noise Consideration}

In a real HDD servo loop, disturbances and sensor noise are inevitably present and may cause the $\mathrm{R} / \mathrm{W}$ head deviate from the desired track center. Hence, they are also impact factors on the quality of written tracks. Disturbances and noise are now considered in the servo loop as shown in Fig. 5. In the block diagram, all disturbances such as disk flutter, vibration, and windage torque are lumped as an equivalent position disturbance $d_{i}(k)$, while measurement noise is denoted by $n_{i}(k)$. Then, the evolution equation have to be derived to incorporate their effects. Assuming that disturbance and noise are independent with each other,

$$
\begin{aligned}
y_{i+1} & =T y_{i}-T u_{i}+T n_{i}+S d_{i} \\
& =T y_{i}-T F e_{i-1}+T n_{i}+S d_{i} \\
& =T y_{i}-T F(P C)^{-1} y_{i}+T F(P C)^{-1} d_{i-1}+T n_{i}+S d_{i} \\
y_{i+1} & =[T-S F] y_{i}+T n_{i}+S d_{i}+S F d_{i-1} .
\end{aligned}
$$

To simplify, $(k)$ 's and $(z)$ 's have been omitted in the equations. According to (12), the track profile of the next 


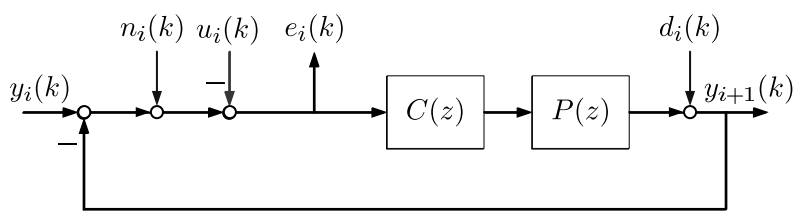

Fig. 5. Block diagram of Self-Servowriting servo loop with disturbance and noise

track $y_{i+1}$ is affected by the disturbance and noise occurred in the current track $\left(d_{i}\right.$ and $\left.n_{i}\right)$ as well as the disturbance in the previous track $\left(d_{i-1}\right)$. The rejection performance on $d_{i}$ and $n_{i}$ has been considered in the design of the feedback controller $C(z)$. The resulting complimentary sensitivity $T(z)$ and sensitivity $S(z)$ provide the specified attenuation properties. On the other hand, from the last term in (12), the rejection ability on $d_{i-1}$ is the combined effect from $S(z)$ and the learning filter $F(z)$. Since the energy of the disturbance usually concentrates at low frequencies, the frequency response of $S(z) F(z)$ should have low gain at these frequencies to attenuate $d_{i-1}$ effectively. In other words, the magnitude of the filter $F(z)$ needs to be constrained such that it will not negate the rejection performance of $S(z)$.

In the design process, an additional condition may be imposed on $F(z)$ to achieve the desired objective. To obtain the condition on magnitude constraint, at first, a weighting function $W(z)$ is designed to be the inverse of some target transfer function of $F(z)$. The goal is to shape the magnitude of $F(z)$ such that $\left|W\left(e^{j \omega}\right) F\left(e^{j \omega}\right)\right|<1$. In the next step, a matrix $\mathbf{W}$ is obtained from the Markov parameters of $W(z)$ such that

$$
\mathbf{W}=\left[\begin{array}{cccc}
w_{0} & 0 & \ldots & 0 \\
w_{1} & w_{0} & \ldots & 0 \\
\vdots & \vdots & \ddots & \vdots \\
w_{N-1} & w_{N-2} & \ldots & w_{0}
\end{array}\right]
$$

Then, the condition is converted to the lifted domain given by

$$
\|\mathbf{W F}\|_{2}=\bar{\sigma}(\mathbf{W F})<1 .
$$

Using similar derivation procedure as in (9), the condition (14) can be formulated as an LMI constraint,

$$
\left[\begin{array}{cc}
\mathbf{I}_{N \times N} & \mathbf{W F} \\
(\mathbf{W F})^{T} & \mathbf{I}_{N \times N}
\end{array}\right]>0 .
$$

Combining (9) and (15), a matrix $\mathbf{F}$ satisfying these two LMI conditions can be solved. Finally, the learning filter $F(z)$ is obtained from the matrix elements of $\mathbf{F}$. In implementation (refer to Fig. 5), the error signal from previous track writing, $e_{i-1}(k)$, is stored in memory units. Next, the compensation signal $u_{i}(k)$ is computed by filtering $e_{i-1}(k)$ through $F(z)$. Then, $u_{i}(k)$ is injected into the servo loop to compensate for $y_{i}(k)$ in order to produce newly written track $y_{i+1}(k)$ with minimum track distortion. The same procedure is repeated in each servowriting step.

\section{SIMULATION STUDY}

In this section, the proposed method is applied to a benchmark problem of hard disk drive control developed by IEEJapan technical committee on Nano-Scale Servo (NSS) system [2006]. The problem was originally for track following mode and is modified to simulate the
SSW process. The simulated drive has 220 sectors with 7200rpm. The disturbance and noise sources used for the simulation consist of disk flutter, windage torque, and white measurement noise. The seed track $y_{0}(k)$ has small non-circularity with standard deviation $\sigma\left(y_{0}\right)$ equal to $1.4 \%$ track width.

\subsection{Without Disturbance and Noise Sources}

Without proper compensation, the written-in error will be propagated and amplified as successive tracks are written causing Radial Error Propagation as shown in Fig. 6. The sigma value, $\sigma\left(y_{i}(k)\right)$, used as a measure of the quality of written tracks, grows rapidly to larger than one track width after 20 tracks even without the existence of disturbance and noise sources. To prevent error propagation, the proposed ILC scheme is employed to the SSW problem. First, the learning filter $F(z)$ is chosen to have five terms with two non-causal coefficients.

$$
F(z)=f_{2}^{N} z^{2}+f_{1}^{N} z^{1}+f_{0}+f_{1}^{C} z^{-1}+f_{2}^{C} z^{-2} .
$$

Then, in order to constrain the magnitude of $F(z)$, the weighting function $W(z)$ in (14) is chosen to be 1 , that means $\mathbf{W}=\mathbf{I}_{N \times N}$ in (13). Finally, a solution $\mathbf{F}$ satisfying two LMI conditions (9) and (15) is found by using the LMI solver (Sturm et al. [2006]). The obtained learning filter $F(z)$ is shown in Fig. 7. Also, the essential transfer function $[T(z)-S(z) F(z)]$ in the evolution equation (3) is shown in Fig. 8. Notice that the resulting maximum gain of $[T(z)-S(z) F(z)]$ is less than 1 . This condition ensures that the effect of non-circular seed track is diminishing with every iteration. Applying the compensation scheme in Fig. 4 with the result shown Fig. 10, it is observed that the sigma value of $y_{i}$ keeps deceasing as track number $i$ increases. In other words, the designed SSW process converges monotonically.

\subsection{With Disturbance and Noise Sources}

Disturbance and noise effect is then considered in the following simulation. The measurement noise $n_{i}$ is modeled as a white noise with a sigma value of $1.5 \%$ track width. The power spectrum density of the equivalent position disturbance $d_{i}$ is shown in Fig. 11. It can be seen that the energy of the disturbance mainly dominates at low

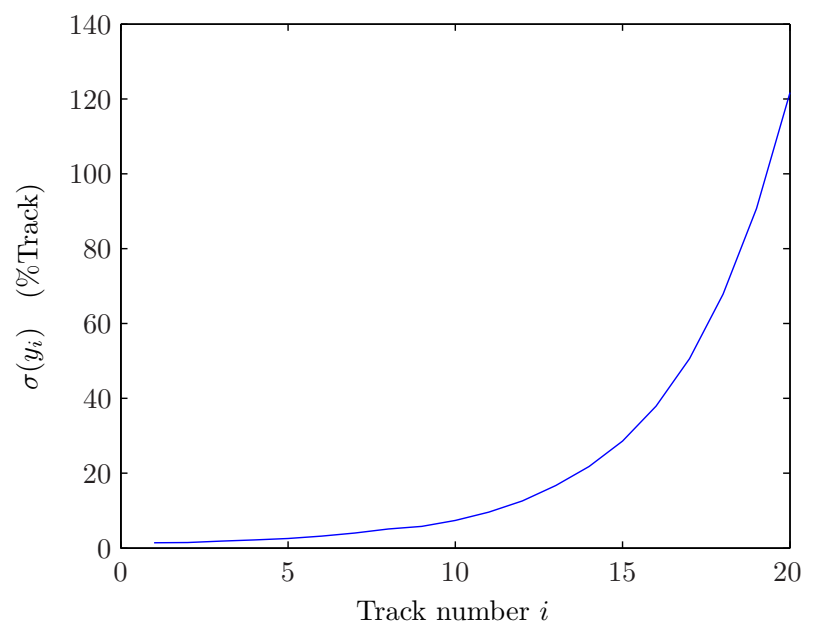

Fig. 6. WIRRO $\left(y_{i}\right)$ without compensation 
Learning filter $F(z)$
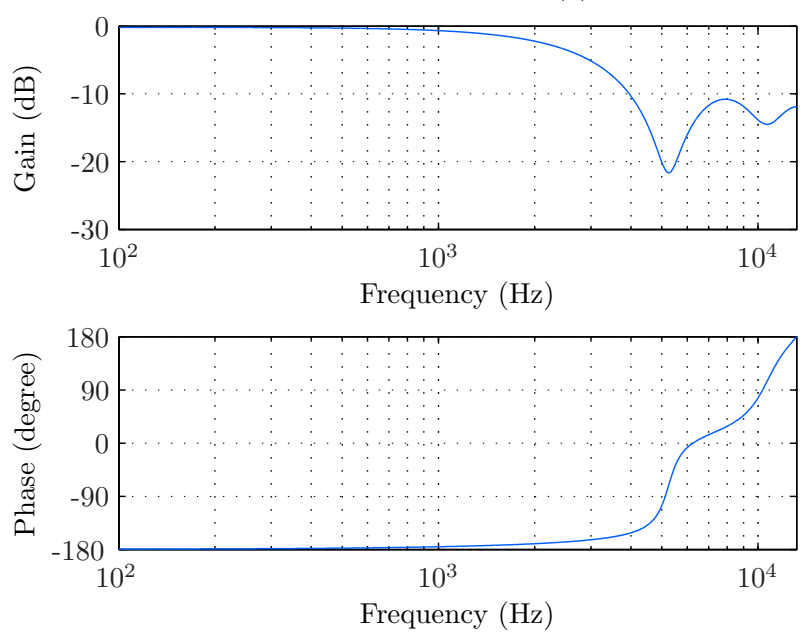

Fig. 7. Frequency response of the designed learning filter $F(z)$

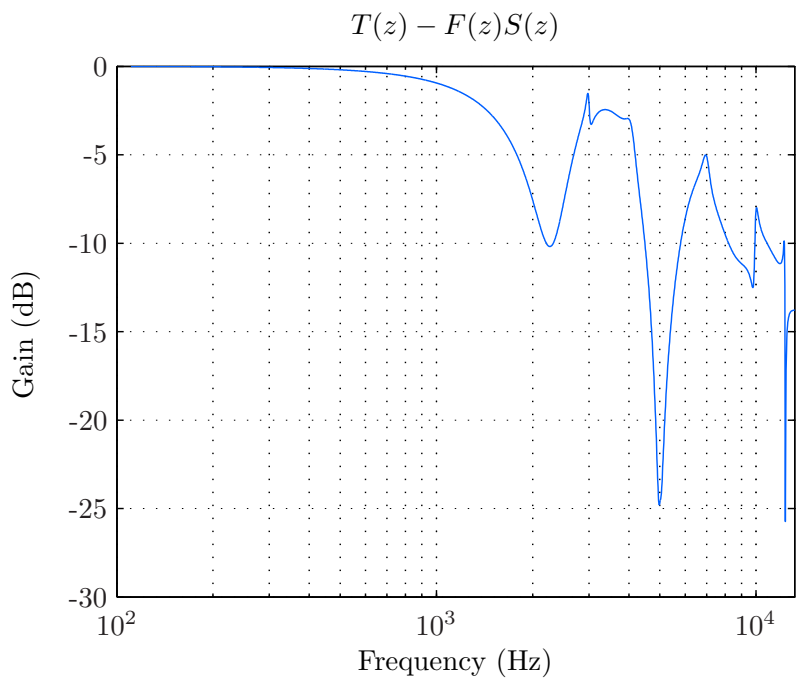

Fig. 8. Frequency response of $T(z)-S(z) F(z)$

frequencies. Now the frequency response of $S(z) F(z)$ needs to be examined, since it affects the performance of disturbance rejection. From Fig. 9, $S(z) F(z)$ preserves the disturbance attenuation property of the sensitivity $S(z)$ at low frequencies due to the low-pass nature and the constrained gain of the learning filter $F(z)$. The sigma values of the first one thousand self servo-written tracks is depicted in Fig. 12. It is observed that the radial error propagation is well contained. Notice that $\sigma\left(y_{i}(k)\right)$ fluctuates as the track propagation is proceeding due to the randomness of the disturbance and noise sources, and that the resulting average $\sigma\left(y_{i}(k)\right)$ of the written tracks is kept at $2.88 \%$ track width. Another common performance index to quantify the quality of written tracks is the minimum spacing between two adjacent tracks on the same sector, also called AC Squeeze. Here, the AC squeeze for track $i$, denoted by $\mathrm{ACsqz}_{i}$ is defined as

$$
\mathrm{ACsqz}_{i}=\min _{k \in(0, N-1)} 1+y_{i}(k)-y_{i-1}(k)
$$

where $y_{i}(k)$ and $y_{i-1}(k)$ are normalized by the track width. The ideal value of $\mathrm{AC}$ squeeze is 1 track width. When the $\mathrm{AC}$ squeeze value is too small, two adjacent tracks with

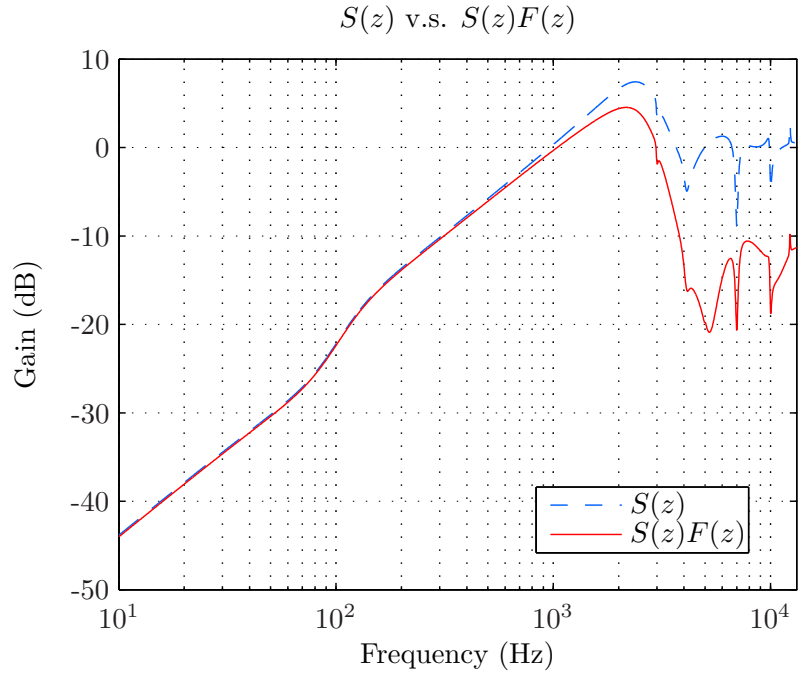

Fig. 9. Frequency response of $S(z)$ and $S(z) F(z)$

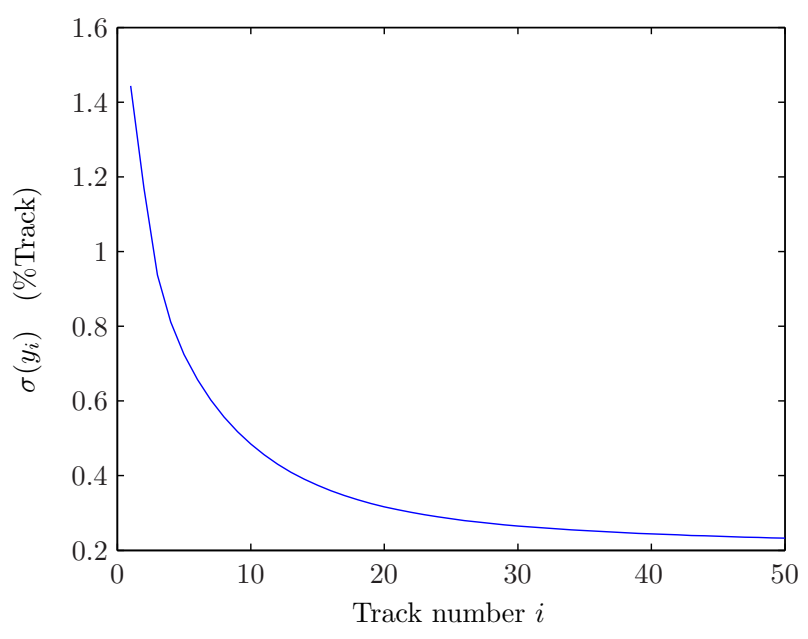

Fig. 10. WIRRO $\left(y_{i}\right)$ with compensation (no disturbance and noise in the loop)

narrow track spacing may interfere with each other and cause data corruption. The histogram of the resulting AC squeeze with an average 0.88 track width is shown in Fig. 13. To sum up, after compensation, the WIRRO and AC squeeze are within acceptable level. Thus, good quality servo tracks are produced with the proposed ILC scheme for SSW process.

\section{CONCLUSIONS}

This paper discussed the self-servowriting process in HDDs. The major challenge is to prevent the written-in errors from propagating across the radial direction in the process. An ILC structure was applied to mitigate the error propagation effect. The disturbance and noise rejection was also considered in the design framework. The proposed LMI-based synthesis scheme provides a systematic design approach. Simulation results have demonstrated that the radial error propagation was contained which results in good quality written tracks with small track distortion and track squeeze. 


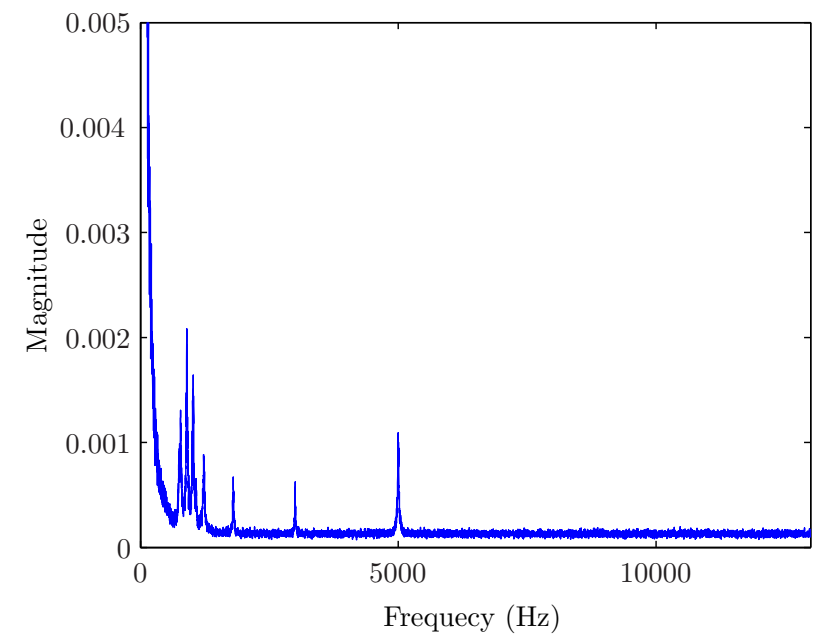

Fig. 11. Power spectral density of the disturbance source

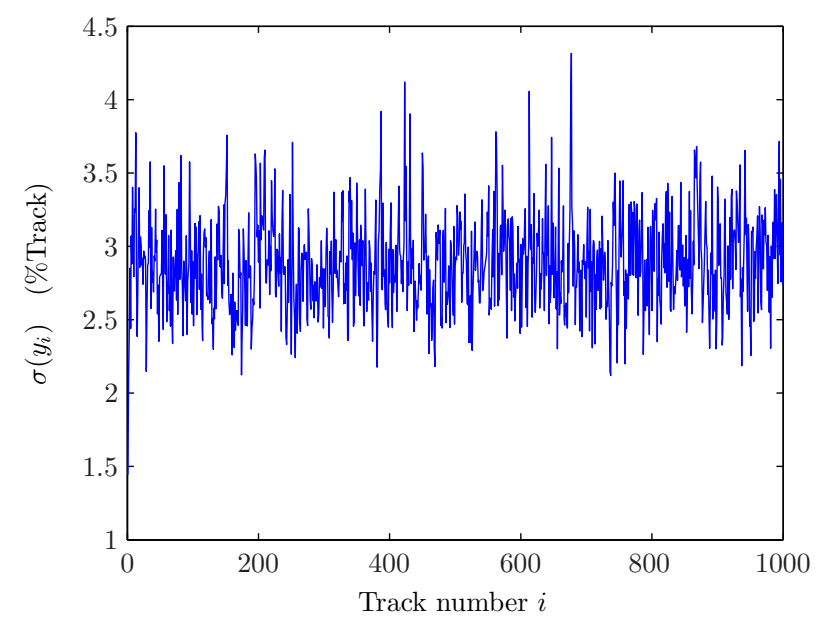

Fig. 12. WIRRO $\left(y_{i}\right)$ with compensation in the presence of disturbance and noise

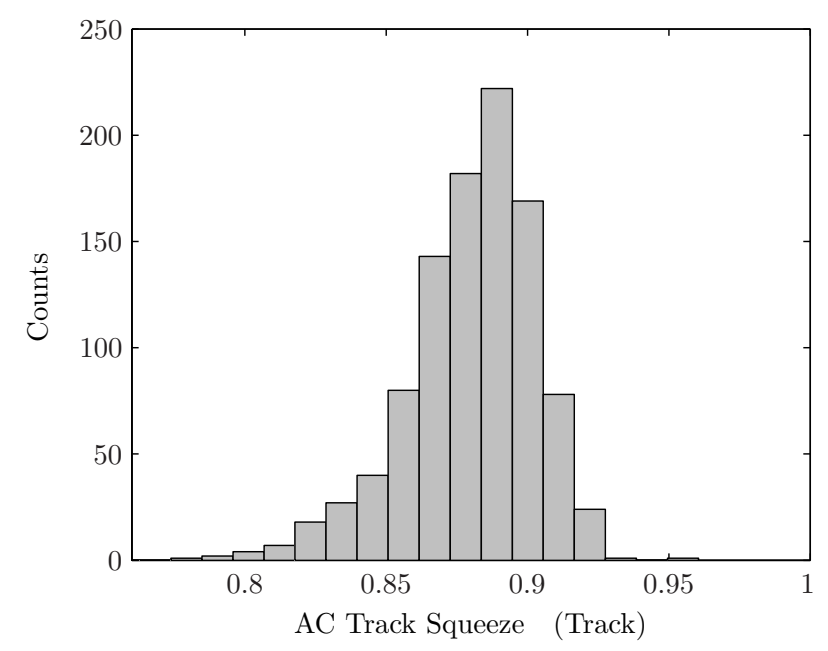

Fig. 13. Histogram of AC track squeeze with compensation

\section{REFERENCES}

A. Al-Mamun, G. Guo, and C. Bi. Hard Disk Drive: Mechatronics And Control. Taylor \& Francis Group, 2007.

S. Boyd and L. Vandenberghe. Convex Optimization. Taylor \& Francis Cambridge University Press, 2004.

D.A. Bristow, M. Tharayil, and A.G. Alleyne. A survey of iterative learning control. IEEE Control Systems Magazine, 26(3):96-114, 2006.

D.F. Cribbs, M.L. Ellenberger, and J.W. Hassler Jr. Self-servowriting disk drive and method. U.S. Patent 5448429, 1995.

IEEJapan technical committee on Nano-Scale Servo (NSS) system. NSS homepage, 2006. URL http://mizugaki.iis.u-tokyo.ac.jp/nss/.

H. Melkote and R.J. McNab. Modeling and control for self-servowriting in hard disk drives: A repetitive process approach. In Proceedings of the 2006 American Control Conference, pages 2005-2010, 2006.

J.F. Sturm, O. Romanko, and I. Pólik. SeDuMi 1.1: a Self-Dual-Minimization package, 2006. URL http://sedumi.mcmaster.ca/.

H. Ye, V. Sng, C. Du, J. Zhang, and G. Guo. Radial error propagation issues in self servo track writing technology. IEEE Transactions on Magnetics, 38(5): 2180-2182, 2002. 